\title{
Compact rectangular waveguide to HMSIW transition
}

\author{
Jun Dong ${ }^{1 \mathrm{a})}$, Yihong Zhou ${ }^{2}$, Tao Yang ${ }^{1}$, and Haiyan Jin $^{3}$ \\ ${ }^{1}$ School of Electronic Engineering, University of Electronic Science and Technology \\ of China, No.2006, Xiyuan Ave, West Hi-Tech Zone, Chengdu, 611731, China \\ ${ }^{2}$ School of Physics and Electronic, University of Electronic Science and Technology \\ of China, Jianshe Road, Chengdu, 610054, China \\ ${ }^{3}$ School of Communication and Information Engineering, University of Electronic \\ Science and Technology of China, No. 4, Section 2, North Jianshe Road, \\ Chengdu, 610054, China
}

a) jundong.ee@gmail.com

\begin{abstract}
A compact waveguide-to-half mode substrate integrated waveguide (HMSIW) transition using antisymmetric tapered probes is proposed. A good matching of field and impendence is achieved by the tapered probes. To verify the proposed transition, a back-to-back test module at Ka-band is fabricated and measured. It provides a return loss better than $14 \mathrm{~dB}$ and an insertion loss of 0.35 to $1 \mathrm{~dB}$ within a frequency range from 25 to $40 \mathrm{GHz}$. There is no need of intermediate transition for this design. The size of the proposed transition is reduced by approximately $83.8 \%$ as compared with the waveguide-to-HMSIW transition using antipodal fin-line.
\end{abstract}

Keywords: compact transition, HMSIW, rectangular waveguide, antisymmetric tapered probes

Classification: Microwave and millimeter wave devices, circuits, and systems

\section{References}

[1] M. Bozzi, A. Georgiadis and K. Wu: IET Microwave Antenn. Propag. 5 (2011) 909. DOI:10.1049/iet-map.2010.0463

[2] W. Hong, B. Liu, Y. Wang, Q. Lai, H. Tang, X. X. Yin, Y. D. Dong, Y. Zhang and K. Wu: IRMMW-THz (2006) 219. DOI:10.1109/ICIMW.2006. 368427

[3] Q. H. Lai, C. Fumeaux, W. Hong and R. Vahldieck: IEEE Trans. Microw. Theory Tech. 57 (2009) 1996. DOI:10.1109/TMTT.2009.2025429

[4] M.-H. Ho and C.-S. Li: IEEE Microw. Wirel. Compon. Lett. 23 (2013) 78. DOI:10.1109/LMWC.2013.2238911

[5] D. E. Senior, X. Y. Cheng and Y.-K. Yoon: IEEE Microw. Wirel. Compon. Lett. 22 (2012) 123. DOI:10.1109/LMWC.2012.2183860

[6] Y.-D. Dong, W. Hong, Z.-Q. Kuai, Y. Chen, Y. Zhang, J. Y. Zhou and J.-X. Chen: IEEE Trans. Antenn. Propag. 56 (2008) 2894. DOI:10.1109/TAP.2008. 928792

[7] L. Xia, R. Xu, B. Yan, J. Li, Y. Guo and J. Wang: Electron. Lett. 42 (2006) 
1403. DOI:10.1049/el:20062228

[8] J. Li, G. Wen and F. Xiao: Electron. Lett. 46 (2010) 223. DOI:10.1049/el. 2010.2518

[9] R. Głogowski, J.-F. Zürcher, C. Peixeiro and J. R. Mosig: Electron. Lett. 49 (2013) 602. DOI:10.1049/el.2013.0388

[10] C. L. Zhong, J. Xu, Z. Y. Zhi and C. X. Jin: Electron. Lett. 45 (2009) 168. DOI:10.1049/el:20092315

\section{Introduction}

Recently, Substrate integrated waveguide (SIW) is a promising candidate for microwave and millimeter-wave applications for its excellent features such as compact size, low loss and easy integration with planar circuit [1]. Half mode substrate integrated waveguide (HMSIW) is a type of miniaturized SIW, which keeps the advantages of SIW but the size is nearly half reduced [2, 3]. Based on HMSIW technology, many kinds of microwave and millimeter-wave components are designed $[4,5,6]$. In spite of the wide use of printed planar circuits in microwave and millimeter-wave systems, rectangular waveguides still play an essential role in various microwave/millimeter-wave systems because of their high performance in terms of quality factor and powerhandling capacity. Therefore, the development of a low insertion loss broadband transition between the rectangular waveguide and planar transmission line is of critical importance. Various transitions between rectangular waveguide and SIW have been reported in open literature [7, 8, 9]. A radial probe is adopted in the design of waveguide to SIW transition [7]. However, the insertion loss of this transition is big and the fabrication of height-tapered waveguide is a little more complex than the standard rectangular waveguide. The antipodal fin-line transition is another one capable of achieving broadband design [8], but suffers from electrically large substrate size. Recently, an aperture-resonance approach on the end of waveguide has also been proposed for easy integration between waveguide and SIW, but generally only $6.6 \%$

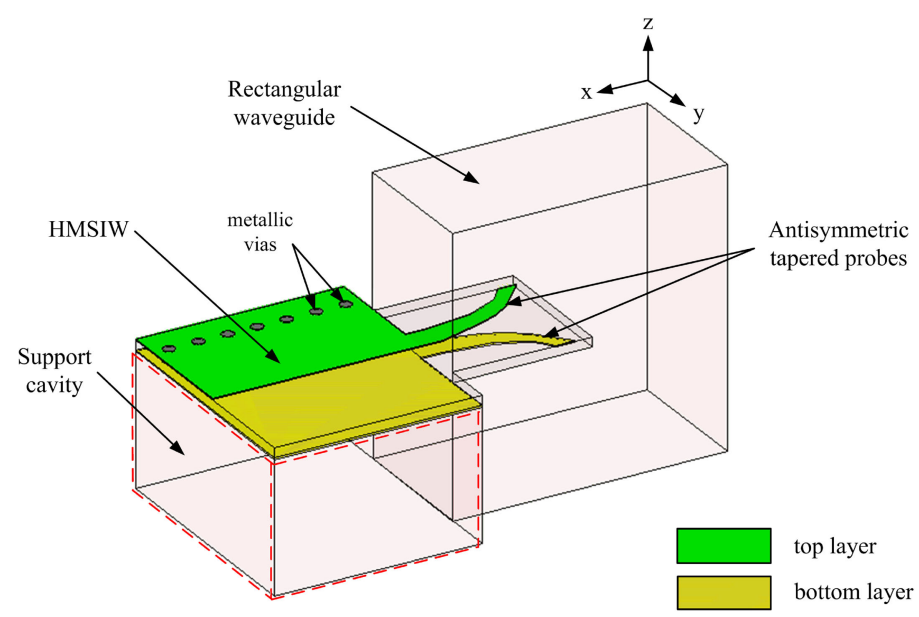

Fig. 1. Structure of waveguide-to-HMSIW transition using antisymmetric tapered probes 
bandwidth can be achieved [9]. A broad rectangular waveguide to HMSIW transition is firstly implemented by using an antipodal fin-line [10]. Although the bandwidth of such transition is wide enough for uses, the circuit size is also large owing to the length of the short-circuited tapered fin-line. Furthermore, an intermediate transition between HMSIW and antipodal fin-line is needed for this transition, which resulting in the transition size increasing and design complexity. The bulky size of this transition limits the use of HMSIW in compact millimeter-wave circuits. It may not meet the requirement of compact and broadband in some millimeter-wave systems.

This work presents a new type of waveguide-to-HMSIW transition based on E-plane probe coupling, as shown in Fig. 1. Two antisymmetric tapered probes are employed to transform the $\mathrm{TE}_{10}$ mode in the rectangular waveguide to the quasi- $\mathrm{TE}_{0.5,0}$ mode in the HMSIW directly. The circuit size of the transition reduced largely since there is no need of an intermediate transition. Compared with the Waveguide-to-HMSIW transition using the antipodal finline [10], the circuit size of the proposed transition is reduced more than $83.8 \%$. In addition to the compact size, the transition is shown to operate well over the entire recommended waveguide band. A back-to-back transition prototype at Ka-band has been fabricated and the scattering parameters are measured to verify the proposed design.

\section{Proposed transition structure}

The structure of the proposed transition is shown in Fig. 1. It can be seen that the transition structure consists of three parts, which are the rectangular waveguide, the antisymmetric tapered probes and the HMSIW. The HMSIW is fabricated by implementing a row of metallic vias on a substrate with metal coating on both sides. The antisymmetric tapered probes printed on opposite sides of the substrate which extended from the end of HMSIW and inserted into the centre of E-plane of a waveguide. The waveguide is a standard WR28 rectangular waveguide.
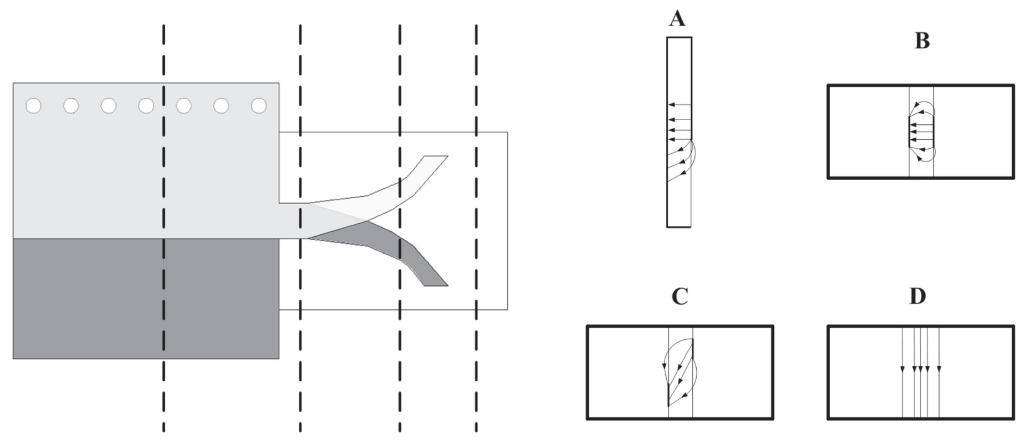

Fig. 2. The E-field matching for waveguide to HMSIW transition

Design of a transmission-line transition requires simultaneous matching of 
matching by antisymmetric tapered probes. Due to the large ratio of HMSIW width to height and the discrete arrangement of the metallic vias, only the quasi- $\mathrm{TE}_{\mathrm{p}-0.5,0}(\mathrm{p}=1,2, \ldots)$ modes can propagate in the HMSIW and the dominant mode is the $\mathrm{TE}_{0.5,0}$ mode [3]. The two probes tapered on opposite sides of the substrate tapered in an antisymmetric structure, which employ spline curve to improve impedance matching with the rectangular waveguide. A progressive change between the high impedance of the waveguide and the low impedance of the HMSIW transmission line can be achieved by properly choosing the dimension of the tapered probes. The antisymmetric tapered probes are placed at the E-plane of the rectangular waveguide, which not only act as an impedance transformer, but also change the y-polarised electric fields of the $\mathrm{TE}_{10}$ mode in the waveguide to the z-polarised electric fields of the quasi- $\mathrm{TE}_{0.5,0}$ mode in the HMSIW smoothly. The transition structure for E-field matching between waveguide and HMSIW is shown in Fig. 2.

\section{Simulation and measurement}

The proposed transition is simulated and optimized using Ansoft high-frequency structure simulator (Ansoft HFSS). The waveguide is a standard WR28 with dimensions of $7.112 \times 3.556 \mathrm{~mm}$, which will ensure single $\mathrm{TE}_{10}$ mode propagation at Ka-band. The antisymmetric tapered probes and HMSIW are integrated into a single RT/Duroid 5880 substrate with relative dielectric constant of 2.22, loss tangent of 0.0009 and thickness of $0.254 \mathrm{~mm}$. The planar circuit of waveguide to HMSIW transition is shown in Fig. 3. The design parameters of the proposed transition circuit are achieved by optimization. The key dimensions of transition circuit are $\mathrm{r}=0.2 \mathrm{~mm}, \mathrm{p}=0.8 \mathrm{~mm}, \mathrm{~W} 0=$ $0.7 \mathrm{~mm}, \mathrm{~W} 1=0.5 \mathrm{~mm}, \mathrm{~W} 2=0.45 \mathrm{~mm}, \mathrm{~W}=0.95 \mathrm{~mm}$, the width of narrow wall of rectangular waveguide is $b=3.556 \mathrm{~mm}$, the width of broad wall of HMSIW is W_hmsiw $=2.6 \mathrm{~mm}$. The whole length of the tapered probes is

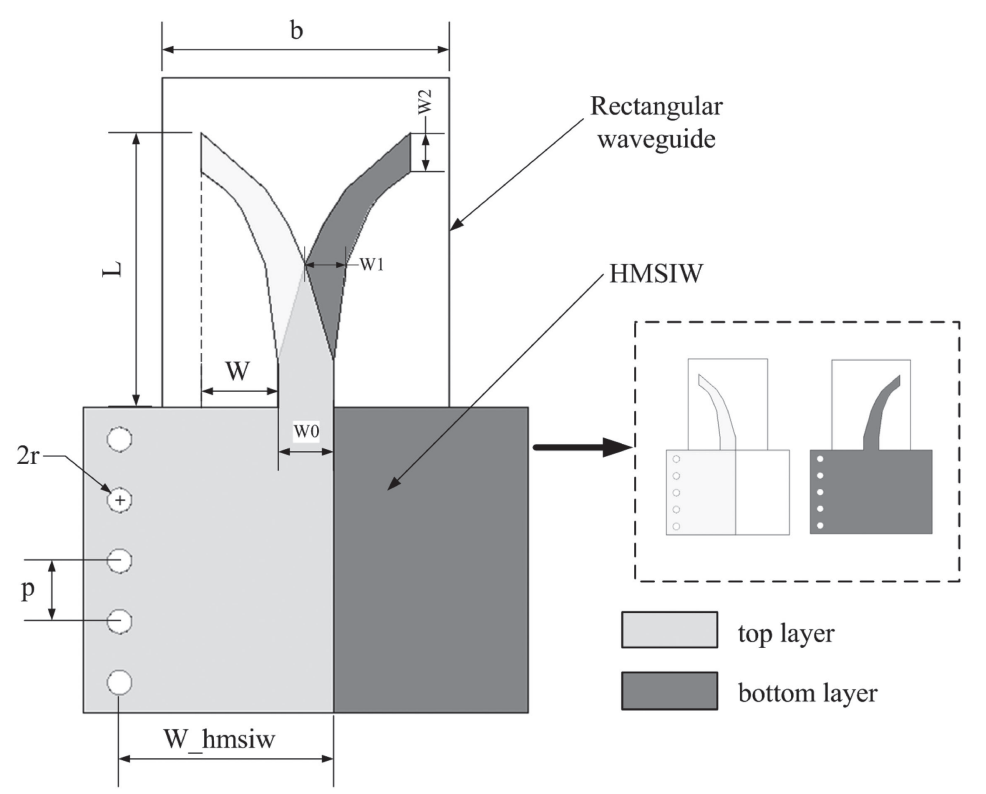

Fig. 3. View of planar circuit for waveguide to HMSIW transition 
$\mathrm{L}=3.38 \mathrm{~mm}$. The whole length for the probe substrate is set to $3.56 \mathrm{~mm}$, which is equivalent to the width of narrow wall of WR-28 waveguide. Compared with the transition using antipodal fin-line [10], the transition length is reduced from 22 to $3.56 \mathrm{~mm}$, which means more than $83.7 \%$ reduction in circuit size. It demonstrated that the circuit size of the proposed transition is reduced largely.

To experimentally test the performance of the proposed transition circuit two identical transitions were cascaded back-to-back. As shown in Fig. 4, a configuration of back-to-back transition was fabricated. The measurement was carried out by using a vector network analyzer after the equipment was calibrated with waveguide calibration kit. The measured results of the backto-back transition are shown in Fig. 5 along with the simulation results obtained using the software HFSS. As can be seen from Fig. 5, the measured and simulated results are in good agreement in the Ka-band except for a small shift in return loss, which may attributed to the deviation in PCB and splitblock cavity fabrication. The measured data shows a better than $14 \mathrm{~dB}$ return

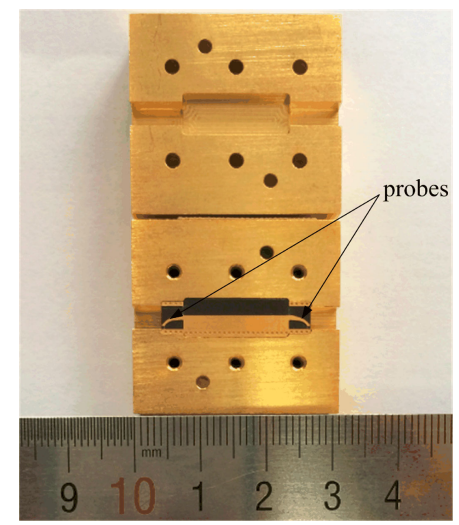

Fig. 4. Photograph of the fabricated back-to-back transition

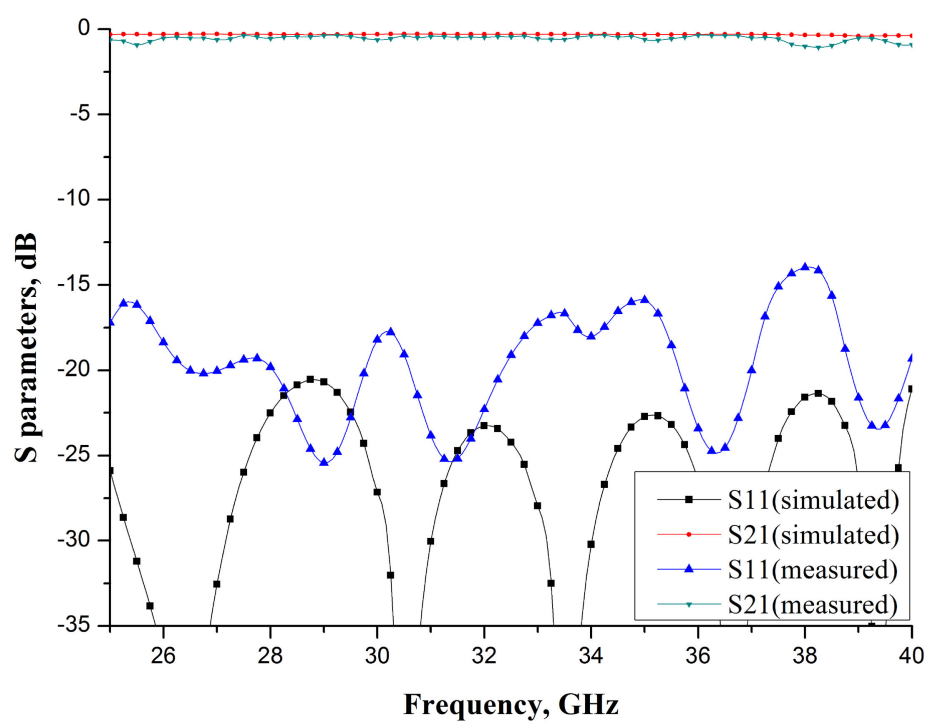

Fig. 5. The simulated and measured results of the back-toback transition 
loss from 25 to $40 \mathrm{GHz}$. The measured insertion loss of the back-to-back transition, including the loss of a $17 \mathrm{~mm}$ HMSIW transmission line, is between 0.35 to $1 \mathrm{~dB}$ within the frequency range of $25-40 \mathrm{GHz}$. Therefore, the loss of a single transition is less than $0.5 \mathrm{~dB}$.

\section{Conclustion}

In this work, a novel compact waveguide-to-HMSIW transition using the antisymmetric tapered probes is proposed. By a gradual change of the tapered probes, a good E-field matching between the waveguide and HMSIW is achieved. There is no need of intermediate transition for this design. To verify the simulation result, a back-to-back transition prototype is fabricated and measured at Ka band. Reasonable agreements between the simulated and measured results are obtained. This work shows a comparable performance for the transitions while having a compact transition circuit. With its compact size and broadband performance, such a transition is suitable for various millimeter-wave applications.

\section{Acknowledgments}

This work was supported by the Fundamental Research Funds for the Central Universities of China (Grant No. ZYGX2012J030 and ZYGX2013J059)). 